\title{
Colonoscopy detects significantly more flat adenomas than 3-tesla magnetic resonance colonography: a pilot trial
}

Authors

Institutions
Robert Hüneburg ${ }^{1, ~ *}$, Guido Kukuk ${ }^{2,}$, , Jacob Nattermann ${ }^{1}$, Christoph Endler ${ }^{1}$, Arndt-Hendrik Penner ${ }^{2}$, Karsten Wolter ${ }^{2}$, Hans Schild ${ }^{2}$, Christian Strassburg ${ }^{1}$, Tilman Sauerbruch ${ }^{1}$, Volker Schmitz ${ }^{1,}{ }^{* *}$, Winfried Willinek ${ }^{2, * *}$

${ }^{1}$ Department of Internal Medicine I, University Hospital of Bonn, Bonn, Germany
2 Department of Radiology, University Hospital of Bonn, Bonn, Germany submitted 21. August 2015 accepted after revision 9. December 2015

\section{Bibliography}

DOI http://dx.doi.org/

10.1055/s-0041-111501

Published online: 28.1.2016

Endoscopy International Open 2016; 04: E164-E169

(c) Georg Thieme Verlag KG Stuttgart · New York

E-ISSN 2196-9736

\section{Corresponding author}

Robert Hüneburg, MD

Department of Internal

Medicine I

University of Bonn

Sigmund-Freud Straße 25

D-53115 Bonn

Germany

Fax: +49-228-2871-9638 robert.hueneburg@ukb.unibonn.de
Background and study aims: Colorectal cancer (CRC) is one of the most common cancers worldwide, and several efforts have been made to reduce its occurrence or severity. Although colonoscopy is considered the gold standard in CRC prevention, it has its disadvantages: missed lesions, bleeding, and perforation. Furthermore, a high number of patients undergo this procedure even though no polyps are detected. Therefore, an initial screening examination may be warranted. Our aim was to compare the adenoma detection rate of magnetic resonance colonography (MRC) with that of optical colonoscopy.

Patients and methods: A total of 25 patients with an intermediate risk for CRC (17 men, 8 women; mean age 57.6, standard deviation 11) underwent MRC with a 3.0-tesla magnet, followed by colonoscopy. The endoscopist was initially blinded to the results of MRC and unblinded immediately after examining the distal rectum. Following

\section{Introduction}

\section{$\nabla$}

Colorectal cancer (CRC) is one of the most common cancers worldwide. It has been shown that colonoscopy reduces CRC-related mortality by up to $50 \%[1,2]$. Therefore, colonoscopy is the current gold standard for the screening and prevention of CRC.

Despite being the appropriate diagnostic and therapeutic tool, colonoscopy nonetheless has disadvantages. First of all, not all relevant lesions are detected. In several back-to-back studies of colonoscopy, up to $22 \%$ of all adenomas were missed during examination [3].

According to epidemiological data, the prevalence of colorectal neoplasia in examined patients in the screening population is approximately onethird, so that $63.5 \%$ to $84 \%$ are without any neo-

\section{License terms

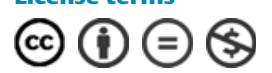

* These authors contributed equally.

** These authors contributed equally. endoscopic excision, the size, anatomical localization, and appearance of all polyps were described according to the Paris classification.

Results: A total of 93 lesions were detected during colonoscopy. These included a malignant infiltration of the transverse colon due to gastric cancer in 1 patient, 28 adenomas in 10 patients, 19 hyperplastic polyps in 9 patients, and 45 non-neoplastic lesions. In 5 patients, no lesion was detected. MRC detected significantly fewer lesions: 1 adenoma $(P=0.001)$ and 1 hyperplastic polyp $(P=0.004)$. The malignant infiltration was seen with both modalities. Of the 28 adenomas, 23 ( $82 \%$ ) were $5 \mathrm{~mm}$ or smaller; only 4 adenomas $10 \mathrm{~mm}$ or larger $(14 \%)$ were detected.

Conclusion: MRC does not detect adenomas sufficiently independently of the location of the lesion. Even advanced lesions were missed. Therefore, colonoscopy should still be considered the current gold standard, even for diagnostic purposes.

plastic lesion. In retrospect, colonoscopy is unnecessary in these persons who have no findings that warrant further diagnosis or treatment [4]. Although colonoscopy is a safely established medical examination, it is not free of risks because of its invasive character [5]. Therefore, it is reasonable to seek additional, alternative screening methods that would allow the preselection of persons most likely to benefit from undergoing colonoscopy. In the United States, computed tomographic colonography (CTC) has been recommended as an alternative screening method by the American Society of Gastrointestinal Endoscopy [6]. However, because of the radiation exposure, it is not generally accepted for screening purposes. As an alternative to CTC, magnetic resonance colonography (MRC) has been proposed for CRC screening. In several studies, MRC was shown to be able to detect large polyps ( $>10 \mathrm{~mm}$ ) with a sensitivity of $84 \%$. The data for small polyps are heterogeneous. All data available at the beginning 
Table 1 Sequence parameters for magnetic resonance colonography (MRC) in a study comparing MRC with colonoscopy for the detection of flat adenomas.

\begin{tabular}{|c|c|c|c|c|c|c|c|}
\hline Sequence & $\mathrm{TR}, \mathrm{ms}$ & $\mathrm{TE}, \mathrm{ms}$ & $\mathrm{FA},{ }^{\circ}$ & Acquisition time, $s$ & Slices, $\mathbf{n}$ & Acquisition voxel, $\mathrm{mm}^{3}$ & FOV, $\mathrm{mm}^{2}$ \\
\hline 3DT1 cor & 3.5 & 1.7 & 10 & 13.3 & 80 & $1.5 \times 1.5 \times 2.3$ & 450 \\
\hline 3DT1 ax & 3.5 & 1.7 & 10 & $2 \times 18.5$ & 200 & $1.5 \times 1.5 \times 2.3$ & 350 \\
\hline T2 TSE ax & 4817 & 50 & 90 & 9.6 & 40 & $2.0 \times 2.1 \times 4.6$ & 450 \\
\hline
\end{tabular}

TR, time to repetition; TE, echo time; FA, flip angle; FOV, field of view; 3D, three-dimensional; T1, spin lattice relaxation time; cor, coronal; ax, axial; T2, spin spin relaxation time; TSE, turbo spin echo.

of the study were derived from MRC at only 1.5 tesla (T) [7-13]. MRC at $3 \mathrm{~T}$ has the potential to improve image quality substantially because of the increased signal-to-noise ratio inherent in higher magnetic field strengths. First studies have shown promising results with respect to lesion detection; however, the role of MRC as a screening tool remains unclear [12].

The aim of this prospective study was to compare MRC at 3.0T with conventional colonoscopy with respect to polyp detection in a colon cancer surveillance setting. The study hypothesis was that there would be no significant difference between MRC and colonoscopy in the detection of adenomas.

\section{Patients and methods}

\section{$\nabla$}

The study protocol was approved by an institutional review board, and each participant provided written informed consent. Between February 2010 and February 2012, patients were enrolled in the study. Inclusion criteria were screening colonoscopy, positive occult fecal blood test, unexplained loss of weight, and follow-up after previous polypectomy. Exclusion criteria were prior colonoscopy in the last 6 months, known chronic inflammatory bowel disease, relevant cardiovascular or pulmonary comorbidity, and contraindications to magnetic resonance imaging (MRI). Furthermore, patients with a CRC syndrome, such as familial adenomatous polyposis or hereditary nonpolyposis colorectal cancer, as well as patients with prior colorectal surgery, were excluded.

\section{Study design and endoscopic technique}

For both examinations, the patients were prepared by undergoing lavage with $4 \mathrm{~L}$ of hypertonic polyethylene glycol solution 24 hours before the procedure.

\section{Magnetic resonance colonography}

Image acquisition was performed with a clinical 3.0-T MRI system (Achieva 3.0T TX; Philips Healthcare, Andover, Massachusetts, USA). For bowel distension, 1500 to $2000 \mathrm{~mL}$ of warm tap water was administered via a rectal catheter (Primed Medizintechnik GmbH, Halberstadt, Germany) with hydrostatic pressure (container placed $1 \mathrm{~m}$ above the patient).

All subjects underwent a standardized MRI protocol, including a coronal T2-weighted turbo spin echo (TSE) sequence, a coronal dynamic T1-weighted fat-suppressed gradient echo sequence before and after intravenous contrast medium application, and a transverse T1-weighted fat-suppressed gradient echo sequence after contrast medium application. Gadobutrol (Gadovist; Bayer Vital GmbH, Leverkusen, Germany) was used as the contrast medium in all patients. The dosage was $0.1 \mathrm{mmol}$ per kilogram of body weight injected at $3 \mathrm{~mL} / \mathrm{s}$, followed by a saline flush of $30 \mathrm{~mL}$ at $3 \mathrm{~mL} / \mathrm{s}$, with an automatic power injector (Spectris; Medrad, Warrendale, Pennsylvania, USA).
Fat suppression was achieved by using spectral selection attenuated inversion recovery (SPAIR). The MRC sequence parameters are detailed in $\odot$ Table 1. Bowel distension was assessed with fast coronal T2-weighted TSE sequences. A 20-mg dose of butylscopolamine (Buscopan; Boehringer Ingelheim, Ingelheim, Germany) was administered intravenously before the acquisition of T1-weighted sequences. Coronal dynamic three-dimensional T1-weighted gradient echo sequences were acquired after fluoroscopic triggering in the arterial phase, in the portal venous phase (approximately 45 seconds after bolus detection), and in the late venous phase (approximately 120 seconds after bolus detection). The transverse T1-weighted gradient echo sequence was acquired immediately after the coronal sequences.

Magnetic resonance studies were evaluated by a board-certified radiologist (blinded) with more than 7 years of experience in abdominal MRI examinations of the colon immediately after imaging and before the performance of optical colonoscopy on the same day.

\section{Endoscopy}

Colonoscopy was performed with high definition video endoscopes (Exera II, HDTV-compatible; Olympus, Tokyo, Japan) by two designated endoscopists (J.N. and V.S.). Each of them had performed more than 1000 colonoscopies before this study. The location of all lesions identified during the colonoscopy that differed from normal mucosa was documented by an independent observer present during the endoscopic examination. All suspicious lesions were documented by describing the anatomical site, distance to the anal margin, appearance, and size as measured with biopsy forceps.

Patients requesting sedation received intravenous midazolam $(1-10 \mathrm{mg})$ or propofol $(20-30 \mathrm{mg})$ before intubation of the colonoscope. Antispasmodic medication (butylscopolamine) was given at the discretion of the endoscopist during the procedure, specifically after the cecum had been reached for mucosal inspection during withdrawal. Further doses of intravenous medication were administered as clinically required.

Insertion and withdrawal times were documented. Measurement of the withdrawal time was started as soon as examination of the cecum began and was stopped when the scope was withdrawn from the anus. Measurement of the withdrawal time was also interrupted whenever a polyp was identified until the polyp had been retrieved and removed, and time measurement was continued as soon as the examination restarted. The same procedure was applied for collecting biopsy specimens. Thus, the measured withdrawal time accurately reflected the time spent for mucosal inspection and did not include any time spent for therapeutic manipulation.

Bowel preparation for the colonoscopic examinations was the same as for the MRI examinations as patients underwent the two examinations within a short time, with the MRI examination preceding colonoscopy. The quality of bowel preparation was documented as very good (100\% mucosal visualization), good 
(> 95\%), fair (between $90 \%$ and $95 \%$ ), or poor (<90\% mucosal visualization). Patients with poor bowel preparation were excluded from the study.

\section{Endoscopic classification of lesions and removal technique}

All lesions identified during colonoscopy either were completely removed by endoscopic biopsy, snare polypectomy, or endoscopic mucosal resection or were biopsied for histopathological examination. In particular, subtle mucosal architectural changes, such as vascular net disruption, discrete mucosal unevenness, and focal pallor or erythema, were documented. Lesions were classified according to the Paris classification by the endoscopist and then documented by the independent observer [14].

Before complete removal of the endoscope, the endoscopist was unblinded regarding the MRC result. Therefore, the endoscopist was able to reexamine the colon in case any polyp that had been detected during MRC before endoscopy was not seen during colonoscopy.

\section{Histopathological analysis}

An expert gastrointestinal pathologist examined all specimens. Tissue was immediately fixed in a $10 \%$ buffered formalin solution and subsequently stained with hematoxylin and eosin. Adenomas were classified according to modified Vienna criteria as either low grade or high grade intraepithelial neoplasia. Invasive neoplasia was defined as neoplastic cellular proliferation extending into submucosal layer 3 or to the muscularis propria.

\section{Statistical analysis}

Statistical differences were analyzed with the paired Student's $t$ test, McNemar's test, or Wilcoxon's signed rank test as appropriate. A two-sided $P$ value of less than 0.05 was considered statistically significant. Calculations were made with SPSS Statistics 22.0 (IBM, Armonk, New York, USA).

The main outcome parameter was the adenoma detection rate. Because no data were available for 3-T MRC before this study, no further case number calculation was possible.

\section{Ethics}

Full ethical approval for the study was granted by the ethics committee of the Medical Faculty, University of Bonn (187/09). Patients were thoroughly informed at least 24 hours before the examination, and informed consent was obtained in writing from every patient before the procedure.

\section{Results}

In total, 32 patients were eligible to take part in the study. Of these, 7 patients had to be excluded, 5 because of poor bowel preparation and 2 because of nonadherence. Thus, a total of 25 patients underwent MRC followed by colonoscopy: 17 men (68\%) and 8 women (32\%) with a mean age (standard deviation [SD]) of 57.6 (11) years. The indications for examination were screening in 10 patients (40\%), follow-up after polypectomy in 6 patients (24\%), positive fecal blood test in 5 patients (20\%), and unexplained loss of weight in 4 patients (16\%). In all 25 patients, the MRC examination was performed adequately. The mean magnetic resonance interpretation time (SD) was 14.2 (1.4) minutes. The colonoscopy examination times were also measured, with the insertion times and withdrawal times measured separately. The mean withdrawal time (SD) was 13.3 (4.7) minutes without polypectomy or snare biopsy.

A total of 93 lesions were detected during colonoscopy. These included a malignant infiltration of the transverse colon due to gastric cancer in 1 patient, 28 adenomas in 10 patients, 19 hyperplastic polyps in 9 patients, and 45 non-neoplastic lesions. In 5 patients no lesions were detected.

MRC detected the malignant infiltration, 1 adenoma (3.6\%), and 1 hyperplastic polyp (5.3\%; 0 Table 2). The locations of the relevant endoscopic findings are displayed in Table 3.

All lesions were classified according to their size. Colonoscopy detected 23 adenomas smaller than $5 \mathrm{~mm}$; one of these was detected by MRC. One adenoma with a size between 6 and $9 \mathrm{~mm}$ was detected by colonoscopy but not detected by MRC. Furthermore, colonoscopy detected 4 adenomas larger than $10 \mathrm{~mm}$ that were not detected by MRC. The exact size and Paris classification of the lesions are shown in $\bullet$ Table 4. Examples of the endoscopic and radiological findings are displayed in $\bullet$ Fig. 1 and $\bullet$ Fig. 2.

\begin{tabular}{|llll|}
\hline & Magnetic resonance colonography & Colonoscopy & P value \\
\hline Examination time, mean (SD), min & $14.2(1.4)$ & $19.2(6.8)$ total & n.s. \\
\hline Adenomas, $\mathrm{n}$ & & $13.3(4.7)$ withdrawal & \\
\hline Patients with $\geq 1$ adenoma, $\mathrm{n}$ & 1 & 28 & 0.001 \\
\hline Hyperplastic polyps, $\mathrm{n}$ & 1 & 10 & 0.001 \\
\hline Malignant infiltration, $\mathrm{n}$ & 1 & 19 & 0.004 \\
\hline
\end{tabular}

Table 2 Results of the two examinations.

n.s., not significant; SD, standard deviation.

Table 3 Location of lesions detected in the study.

\begin{tabular}{|c|c|c|c|c|c|}
\hline & Lesions, $\mathbf{n}$ & Percentage of total, \% & Adenomas & Hyperplastic polyps & Malignant infiltration \\
\hline Cecum & 1 & 2.1 & 1 & 0 & 0 \\
\hline Colon ascendens & 10 & 20.8 & 9 & 1 & 0 \\
\hline Colon transversum & 9 & 18.8 & 7 & 1 & 1 \\
\hline Colon descendens & 4 & 8.3 & 4 & 0 & 0 \\
\hline Sigmoid colon & 10 & 20.8 & 5 & 5 & 0 \\
\hline Rectum & 14 & 29.2 & 2 & 12 & 0 \\
\hline Total & 48 & 100.0 & 28 & 19 & 1 \\
\hline
\end{tabular}


Table 4 Size and appearance of all adenomas $(N=28)$ detected by the two modalities.

\begin{tabular}{|c|c|c|c|c|c|c|c|}
\hline & \multicolumn{2}{|l|}{$\leq 5 \mathrm{~mm}$} & \multicolumn{2}{|l|}{$6-9 \mathrm{~mm}$} & \multicolumn{2}{|l|}{$\geq 10 \mathrm{~mm}$} & \multirow[t]{2}{*}{ Total number of adenomas } \\
\hline & Polypoid & Nonpolypoid & Polypoid & Nonpolypoid & Polypoid & Nonpolypoid & \\
\hline Colonoscopy & 11 & 12 & 0 & 1 & 1 & 3 & 28 \\
\hline MRC & 0 & 1 & 0 & 0 & 0 & 0 & 1 \\
\hline Total & 11 & 12 & 0 & 1 & 1 & 3 & 28 \\
\hline
\end{tabular}

MRC, magnetic resonance colonography.
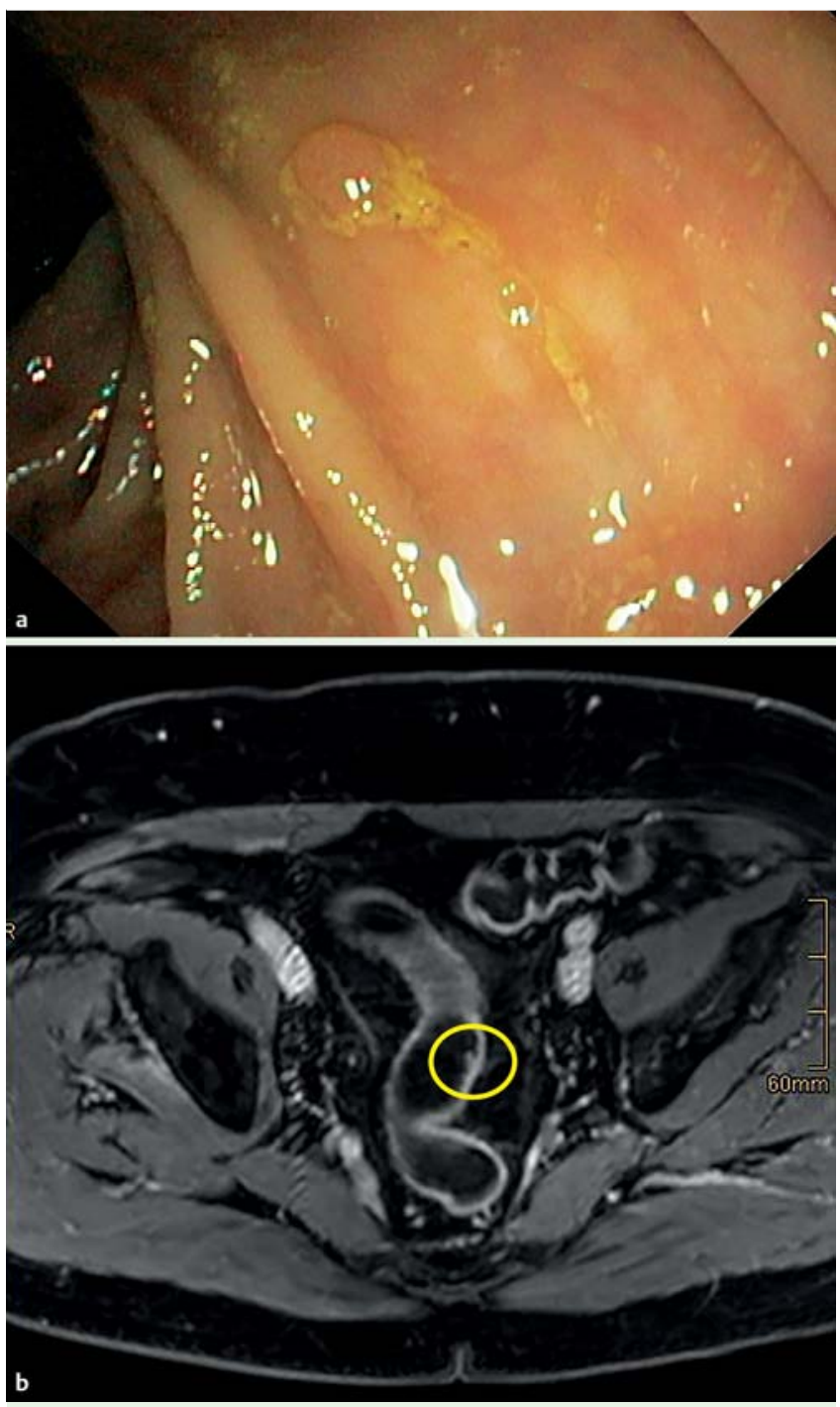

Fig. 1 a Colonoscopy detects a small nonpolypoid tubular adenoma in the sigmoid colon. b Magnetic resonance imaging detects the same lesion (yellow circle).

No additional endoscopic examination was needed because no further lesions were detected during MRC.

Concerning histology, all detected adenomas were tubular adenomas with low grade dysplasia except for 1 lesion with tubulovillous histology. Therefore, neither advanced adenomas nor infiltrative neoplastic lesions were detected, except for a single gastric cancer infiltration into the transverse colon.

Hyperplastic polyps were detected in 9 patients (36\%). Most of them were located in the rectum and sigmoid colon except for 1 in the transverse colon and 1 in the ascending colon.
No complications due to MRC or colonoscopy were reported. Important extracolonic findings were reported in two patients. In one case, a malignant infiltration by gastric cancer was described; in the second, a liver tumor was detected, which was later histologically classified as a cholangiocellular carcinoma.

\section{Discussion}

In our study, colonoscopy detected significantly more adenomas than MRC independently of lesion size or location. The adenoma detection rate by 3-T magnetic resonance tomography in our study was disappointing; however, it corresponded to that in another study, which reported adenoma detection rates of $13 \%$ and $29 \%$ by 3-T MRC and endoscopic colonoscopy, respectively [15]. Several factors may have contributed to this finding. First of all, we reported a high number of small flat lesions by colonoscopy. It is entirely plausible that these lesions would be difficult to detect by magnetic resonance examination, in which intraluminal morphological mucosal alterations are necessary for detection. Of note, the role of flat lesions and the serrated carcinoma pathway in CRC development has only recently gained attention and awareness among endoscopists and may have increased their alertness regarding flat lesions.

Colonoscopy reduces the incidence and associated mortality of CRC [16]. Furthermore, there are plenty of data available underscoring the clinical benefit of removing adenomatous polyps during colonoscopy [1]. With these facts taken together, colonoscopy is the accepted gold standard for the screening and prevention of CRC and has been adopted as part of a nationwide screening program in Germany.

However, there are disadvantages of colonoscopy that must be considered. In several back-to-back studies of colonoscopy, endoscopists missed small ( $\leq 5 \mathrm{~mm}$ ) polyps in up to $28 \%$ and even polyps larger than $1 \mathrm{~cm}$ in up to $8 \%$ of all cases [3]. Efforts have been undertaken to reduce the failure rate of colonoscopy [17].

Screening colonoscopy is also affected by the occurrence of socalled interval cancers, which are cancers that develop despite the performance of adequate screening colonoscopy. This phenomenon compromises the effectiveness of colonoscopy; it is unknown if it is also relevant to other methods-although very plausibly it is - and therefore not singularly attributable to colonoscopy [18].

Major complications like perforation and bleeding occur in up to 2.8 of 1000 examinations [5]. Although a lower incidence of major complications was reported in Germany, this aspect of colonoscopy has to be considered carefully, particularly in a screening setting [19].

Principally, CRC screening targets the following: cancer, adenomas larger than $10 \mathrm{~mm}$, and adenomas with advanced histopathology or a villous component. However, these lesions are de- 

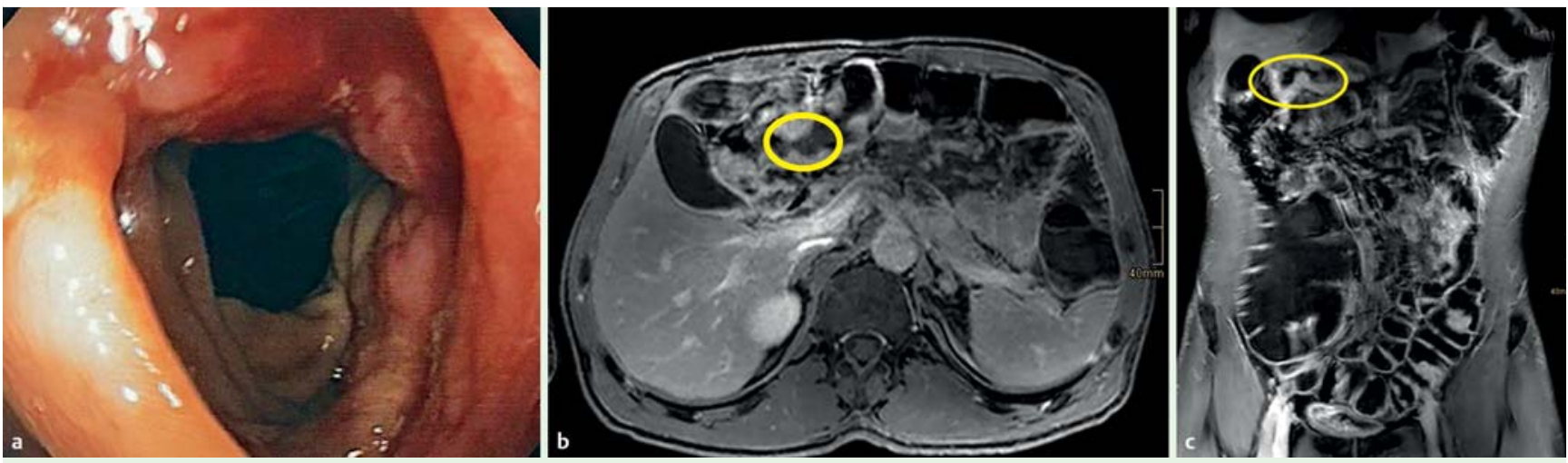

Fig.2 a Colonoscopy detects malignant infiltration of the transverse colon due to gastric cancer. b, c Magnetic resonance imaging (axial view in b, coronal view in c) detects the same lesion (yellow circles).

tected in only $16 \%$ of the screening population; therefore, colonoscopy in retrospect is unnecessary in $84 \%$, with the latter group subjected to unnecessary risks [4]. However, no method is available for detailed sub-stratification of the screening population.

Based on these considerations, alternative methods are of interest, and for this purpose, CTC has been evaluated in several studies [20]. However, the associated radiation burden is a serious disadvantage of this method. MRC at $1.5 \mathrm{~T}$ was evaluated in several studies [7-12] and resulted in satisfactory detection rates for polyps larger than $10 \mathrm{~mm}$. Despite these promising results, it has been implemented neither in current guidelines nor in routine clinical work-up for several reasons, mainly high cost and limited availability. In this study, our aim was to compare 3-T MRC with high definition white-light colonoscopy, which has been shown to be superior to standard white-light endoscopy [21].

Interestingly, current data show that colonoscopy prevents cancer to a smaller extent in the right hemicolon than in the left side of the colon [22,23]. This may be partially due to missed lesions in the right side of the colon. Of note, in our study the highest number of adenomas was detected in the right side of the colon. This may be attributable to the use of high definition endoscopy and an awareness of side differences in prior colonoscopic studies.

Graser et al. reported an adequate detection rate for adenomas larger than $6 \mathrm{~mm}$ and also for advanced neoplasia [15]. Actually, the clinical relevance of small and diminutive polyps or adenomas is still controversial, and adenomas smaller than $6 \mathrm{~mm}$ were considered to be clinically irrelevant in radiological studies in which MRC or CTC was applied $[6,15]$. The crucial point is that when MRC or CTC fails to identify smaller lesions, the risk for cancer in people having 3 or more small adenomas is strongly underestimated; CRC risk is substantially increased in these patients. This risk is present despite the diminutive size of each individual lesion [24,25]. Our study identified 5 patients appearing to be at higher risk on colonoscopy: 1 patient with an adenoma that had tubulovillous histology, 2 patients with 3 adenomas, 1 patient with 4 adenomas, and 1 patient with 7 adenomas, all of which were missed by MRC. This also holds true for the patient with 2 hyperplastic polyps proximal to the sigmoid colon that were detected by colonoscopy. Considering the current insight that these lesions are associated with colorectal neoplasia [26], it was vitally important to detect and eradicate them.
To our surprise, even large adenomas (>10 mm) were missed by MRC. This is of extreme clinical importance because the risk for the development of carcinoma in adenomas larger than $10 \mathrm{~mm}$ is very high [27]. In several multivariate analyses, size, multiplicity, and the presence of a villous component in a polyp appeared to be independent risk factors for advanced neoplasia, in contrast to high grade dysplasia $[28,29]$. The main aim of colonoscopy is to detect advanced neoplasia or cancer. This was not achieved sufficiently by MRC in our study.

One striking advantage of CTC or MRC might be the capacity to detect extracolonic findings, as was the case in our study, in which magnetic resonance tomography revealed cholangiocellular carcinoma and also gastric cancer (with invasion into the transverse colon).

Although our study indicates the superiority of endoscopic colonoscopy in comparison with 3-T MRC, three main limitations have to be considered: the sample size was quite small, this was a single-center study, and the adenoma detection rate of $40 \%$ was relatively high, exceeding data from a general screening population with rates of about $20 \%$ [19]. Although this might be partially attributed to the selection effects of a tertiary medical center, it also might be due to the use of high definition endoscopes and the increasing awareness among endoscopists of the potential for carcinogenesis in flat adenomas.

In conclusion, this study favors endoscopic colonoscopy over MRC for the detection of large and small flat lesions. According to the results we have presented, a major obstacle of MRC seems to be its weakness in detecting small flat lesions, and even adenomas larger than $10 \mathrm{~mm}$. This leaves endoscopic colonoscopy as the standard at this moment. Our results indicate the need for further studies.

\section{Competing interests: None}

\section{References}

1 Zauber AG, Winawer SJ, O'Brien MJ et al. Colonoscopic polypectomy and long-term prevention of colorectal-cancer deaths. N Engl J Med 2012; 366: 687-696

2 Schoen RE, Pinsky PF, Weissfeld JL et al. Colorectal-cancer incidence and mortality with screening flexible sigmoidoscopy. N Engl J Med 2012; 366: $2345-2357$

3 van Rijn JC, Reitsma JB, Stoker J et al. Polyp miss rate determined by tandem colonoscopy: a systematic review. Am J Gastroenterol 2006; 101: $343-350$

4 Regula J, Rupinski M, Kraszewska E et al. Colonoscopy in colorectal-cancer screening for detection of advanced neoplasia. N Engl J Med 2006; 355: 1863-1872 
5 Whitlock EP, Lin JS, Liles $E$ et al. Screening for colorectal cancer: a targeted, updated systematic review for the U.S. Preventive Services Task Force. Ann Intern Med 2008; 149: 638-658

6 Rex DK, Johnson DA, Anderson JC et al. American College of Gastroenterology guidelines for colorectal cancer screening 2008. Am J Gastroenterol 2009; 104: 739-750

7 Leung WK, Lam WWM, Wu JCY et al. Magnetic resonance colonography in the detection of colonic neoplasm in high-risk and average-risk individuals. Am J Gastroenterol 2004; 99: 102 - 108

8 Ajaj W, Pelster G, Treichel U et al. Dark lumen magnetic resonance colonography: comparison with conventional colonoscopy for the detection of colorectal pathology. Gut 2003; 52: $1738-1743$

9 Hartmann D, Bassler B, Schilling D et al. Colorectal polyps: detection with dark-lumen MR colonography versus conventional colonoscopy. Radiology 2006; 238: $143-149$

10 Kuehle CA, Langhorst J, Ladd SC et al. Magnetic resonance colonography without bowel cleansing: a prospective cross sectional study in a screening population. Gut 2007; 56: 1079-1085

11 Florie J, Jensch S, Nievelstein RAJ et al. MR colonography with limited bowel preparation compared with optical colonoscopy in patients at increased risk for colorectal cancer. Radiology 2007; 243: 122 - 131

12 Saar B, Meining A, Beer A et al. Prospective study on bright lumen magnetic resonance colonography in comparison with conventional colonoscopy. Br J Radiol 2007; 80: 235-241

13 Zijta F, Bipat S, Stoker J. Magnetic resonance (MR) colonography in the detection of colorectal lesions: a systematic review of prospective studies. Eur Radiol 2010; 20: 1031 - 1046

14 The Paris endoscopic classification of superficial neoplastic lesions: esophagus, stomach, and colon: November 30 to December 1, 2002. Gastrointest Endosc 2003; 58: S3-S43

15 Graser A, Melzer A, Lindner E et al. Magnetic resonance colonography for the detection of colorectal neoplasia in asymptomatic adults. Gastroenterology 2013; 144: 743 - 750.e742

16 Nishihara $R, W u K$, Lochhead P et al. Long-term colorectal-cancer incidence and mortality after lower endoscopy. N Engl J Med 2013; 369: $1095-1105$

17 Leufkens AM, van Oijen MGH, Vleggaar FP et al. Factors influencing the miss rate of polyps in a back-to-back colonoscopy study. Endoscopy 2012; 44: 470-475
18 Solon JG, Al-Azawi D, Hill A et al. Colonoscopy and computerized tomography scan are not sufficient to localize right-sided colonic lesions accurately. Colorectal Dis 2010; 12: e267-e272

19 Pox CP, Altenhofen L, Brenner $H$ et al. Efficacy of a nationwide screening colonoscopy program for colorectal cancer. Gastroenterology 2012; 142: 1460 - 1467.e1462

20 Pickhardt PJ, Choi JR, Hwang I et al. Computed tomographic virtual colonoscopy to screen for colorectal neoplasia in asymptomatic adults. N Engl J Med 2003; 349: 2191 -2200

21 Tribonias G, Theodoropoulou A, Konstantinidis $K$ et al. Comparison of standard vs high-definition, wide-angle colonoscopy for polyp detection: a randomized controlled trial. Colorectal Dis 2010; 12: e260 e266

22 Brenner $H$, Chang-Claude J, Jansen $L$ et al. Reduced risk of colorectal cancer up to 10 years after screening, surveillance, or diagnostic colonoscopy. Gastroenterology 2014; 146: 709-717

23 Baxter NN, Warren JL, Barrett MJ et al. Association between colonoscopy and colorectal cancer mortality in a US cohort according to site of cancer and colonoscopist specialty. J Clin Oncol 2012; 30: 2664-2669

24 Lieberman DA, Rex DK, Winawer SJ et al. Guidelines for colonoscopy surveillance after screening and polypectomy: a consensus update by the US Multi-Society Task Force on Colorectal Cancer. Gastroenterology 2012; 143: $844-857$

25 Lieberman DA, Weiss DG, Harford WV et al. Five-year colon surveillance after screening colonoscopy. Gastroenterology 2007; 133: 1077 - 1085

26 Schreiner MA, Weiss DG, Lieberman DA. Proximal and large hyperplastic and nondysplastic serrated polyps detected by colonoscopy are associated with neoplasia. Gastroenterology 2010; 139: 1497-1502

27 Heitman SJ, Ronksley PE, Hilsden RJ et al. Prevalence of adenomas and colorectal cancer in average risk individuals: a systematic review and meta-analysis. Clin Gastroenterol Hepatol 2009; 7: 1272-1278

28 Martínez ME, Baron JA, Lieberman DA et al. A pooled analysis of advanced colorectal neoplasia diagnoses after colonoscopic polypectomy. Gastroenterology 2009; 136: 832-841

29 de Jonge $V$, Sint Nicolaas J, van Leerdam ME et al. Systematic literature review and pooled analyses of risk factors for finding adenomas at surveillance colonoscopy. Endoscopy 2011; 43: 560-574 